\title{
Research Paper: \\ Effects of Rhythmic and Simple Auditory Stimulations on Learning the Timing of Sequential Motor Task in Children With DCD
}

\author{
Ahmad Dehnavi $^{1}$ (D), Alireza Saberi Kakhaki ${ }^{{ }^{*}}$ (D) , Hamidreza Taheri Torbati ${ }^{1}$ (D) Mohammadreza Shahabi Kaeb $^{2}$
}

1. Department of Motor Behavior, Faculty of Sport Sciences, Ferdowsi University of Mashhad, Mashhad, Iran.

2. Faculty of Physical Education and Sport Sciences, Hakim Sabzevari University, Sabzevar, Iran.

$\begin{aligned} & \text { Use vour device to scan } \\ & \text { and read the article online }\end{aligned}$
$\begin{aligned} & \text { Citation Dehnavi A, Saberi Kakhaki AR, Taheri Torbati HR, Shahabi Kaeb MR. Effects of Rhythmic and Simple Auditory Stimu- } \\ & \text { lations on Learning the Timing of Sequential Motor Task in Children With DCD Journal of Exercise Science and Medicine. 2020; } \\ & \text { 12(1):25-34. http://dx.doi.org/10.32598/JESM.12.1.3 }\end{aligned}$
doi http://dx.doi.org/10.32598/JESM.12.1.3

\section{(1) (8)}

Article info:

Received: 25 Jul 2019

Accepted: 09 Sep 2019

Available Online: 01 Jan 2020

Keywords:

Acoustic Stimulation, Rhythmic,Sequntial motor task, Motor timing

\section{ABSTRACT}

Introduction: Children and adolescents with Developmental Coordination Disorder (DCD) usually fail to understand spatial awareness and motor timing. The present study assessed Rhythmic Auditory Stimulations (RAS) and Simple Auditory Stimulations (SAS) to facilitate the learning of timing in sequential motor task and recorded the results of their relative and absolute timing errors.

Materials and Methods: 56 male students aged 9-12 years with DCD in Neyshabur city were selected by diagnosing with the following tools: Teacher Motor Inventory, Parental Developmental Coordination Disorder Questionnaire, Movement Assessment Battery for Children-Second (MABC-2 movement test), spatial-visual memory assessment, intelligence assessment, RT test, and biopsychological health status evaluation. Then, they were divided into RAS, SAS, control group 1 (visual), and control group 2. Moreover, a pretest score was performed. Next, the training protocol was presented in 10 blocks of 5 attempts and the acquisition test was performed. Finally, the retention and transfer tests were performed after 48 hours and 10 minutes, respectively.

Results: Based on the obtained results, a significant difference was observed between the visual stimulation group and the RAS and SAS groups in relative and absolute timing components $(\mathrm{P} \leq 0.05)$. However, no significant difference was reported between the rhythmic, SAS, and visual-auditory stimulation groups in relative and absolute timing variables $(P>0.05)$.

Conclusion: Finally, no significant difference was observed between the RAS and simple stimulations although the graphs displayed the effect of the first stimulation more than that of the second one.

\section{* Corresponding Author:} Alireza Saberi Kakhki, PhD.

Address: Department of Motor Behavior, Faculty of Sport Sciences, Ferdowsi University of Mashhad, Mashhad, Iran. Tel: +98 (915) 1689538

E-mail: askakhki@um.ac.ir 


\section{Introduction}

hildren and adolescents with Devel-

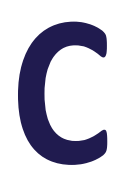
opmental Coordination Disorder (DCD) usually encounter motor problems and act poorer, compared to their healthy counterparts respecting motor skills, despite their relatively normal appearance [1]. DCD, as a chronic neurological disorder, affects more than $6 \%$ to $7 \%$ of school children with motor learning deficits and behavioral coordination disorders [2]. Furthermore, children with DCD require further time to respond to visual signals [3]. Children with DCD fail to understand spatial awareness and motor timing and are less involved in sports and motor activities [1]. Further, children with DCD present a special internal model which affects their ability to move concerning time sequence.

According to some researchers, these problems are due to the lack of production or monitoring of the internal models of action, i.e., called the Internal Modeling Deficit (IMD) hypothesis [3, 4]. Based on this model, the stability of the motor system with a sudden change fails to provide the means for facilitating sensory feedback [5], leading to a delay in the response of the motor pattern [6]. Wilson et al. reported that IMD can be positively affected by rhythmic coordination exercises and timing tasks [7]. The sequence of timing in performing regular tasks requires the integration of the motor cortex, leading to incomplete functions due to lack of bilateral coordination between the cerebral cortex, and the cerebellum of individuals with DCD [8].

Some studies indicated that continuous timing exercises lead to more activation of the motor cortex in an individual with DCD $[6,9]$. Defects in timing perception are probably a major feature among children with DCD. Moreover, the ability of perceptual timing is a prerequisite for controlling their coordination, which seems necessary [2]. However, some studies emphasize the improvement of performance and learning of timing tasks in children with DCD; accordingly, timing can be taught to these children with different protocols $[6,10]$. Thus, some researchers aim to improve learning timing in children and adolescents with DCD by following different protocols and regard the interference of auditory stimulations as one of their executive protocols due to the special characteristics of the human auditory system. This is because the auditory system is very sensitive to time intervals in music and speech. Furthermore, auditory stimulation activates the motor system in the brain even without instructions. Therefore, auditory timing training may be a useful strategy, which can help improve the motor function of individuals with DCD [2]

Based on the evidence, rhythm perception is active in auditory cells and the forehead, Supplementary Motor Area (SMA), basal ganglia, and cerebellum. Accordingly, special circuits in domestic production play a significant role in representing the audio beat structure. Additionally, the cerebellum is involved in the precise and complex encoding of sequences, rapid timing features, and duration [11]. Patel and Iversen indicated that SMA plays an essential role in distance timing. It is considered as the main area in perceiving the rhythm-based time using Action Simulation for Auditory Prediction (ASAP) hypothesis [12]. This auditory beat can be useful for coordination in IMD. Besides, it is regarded as a defect in inverse and forward models stemming from comparing the predicted and real results [2]. Based on other studies, receiving rhythmic temporal information is vaguely related to active auditory involvement [13]. Coordinating with auditory stimulations over visual ones continuously activates the putamen, i.e., a key for metronome and audio beat [14].

The present study aimed to examine whether adding audio beat protocol helps learning timing tasks for children with DCD, or whether the type of auditory stimulation including rhythmic and simple affects this learning.

The basis of count or beat is considered as the speed and rhythm of sound. Moreover, it is the measure of the structural order of each auditory rhythm. Patel and Iversen investigated patients with autism by playing rhythmic music in the laboratory; subsequently, these scholars found that they improved during the step of $12 \%$, walking speed of $25 \%$, and step speed of $10 \%$ [13]. Based on the Neural Resonance theory, to perceive auditory beat, the nervous system draws nonlinear oscillations to match the external auditory stimulation [13]. Normally, human beings can perceive and coordinate most pieces of music between 120 BPM and 150 BPM; thus, information is easily processed.

However, Kral believed that attention energy includes better processing ease when waiting for a beat than for non-listening time [15]. Perceiving the auditory beat is designed in the brain based on the simulation of periodic action in the programming areas of the brain and the two-way signals of these areas with the auditory ones. The Purified Perception of the auditory beat, such as listening in the absence of obvious movement strongly involves the motor system including the pre-motor cortex, the basal ganglia, and the SMA. In other words, there 
is a strong relationship between perceiving the auditory rhythmic element and the various motor functions in the brain [16]. The results of brain functional Magnetic Resonance Imaging (fMRI) in children with DCD indicated a significant difference in patterns of brain activity with other children, especially in the attentional and motor networks. Thus, a new approach should be developed to facilitate the learning of the tasks due to defects in the timing sensor and auditory perception [2]. In addition, Edwards argued that using rhythmic auditory stimulation is regarded as a simplification in the learning process [17]. The present study aimed to explore whether this simplification can be equally effective for children with DCD and whether it is possible to change the learning timing tasks among these children by adding simple and rhythmic auditory stimulations.

\section{Materials and Methods}

The study population included all elementary school students in Neyshabur City, Iran, who were divided into Rhythmic Auditory Stimulation (RAS), Simple Auditory Stimulation (SAS), control group 1, and control group 2, after screening based on the research objectives. The study subjects included 56 right-handed (defined by Edinburgh Inventory test) male students aged 9-12 years, who were diagnosed with DCDQ-7. Using G*power 3.1.9.2 software at $P<0.05$, the confidence level of $90 \%$, and effect size of 0.15 , despite having normal vision, hearing, and intelligence [18].

The children with DCD attended more than 35 boys' elementary schools, including governmental and nongovernmental, i.e., randomly selected in coordination with Neyshabur Education Department. Then, the PMOQ-T form was completed by $>210$ teachers for 6100 students and those suspected of behavioral movements related to DCD were identified. Next, the signed informed consent form was obtained and the DCDQ form was completed by the students' parents. Then, Wechsler's visual-spatial memory and intelligence tests for children (scores above 85 ) were performed by two prominent psychologists in Neyshabur. The inclusion and exclusion criteria of the study were modified when a student was diagnosed with other disorders, such as hyperactivity, autism, and a problem was recorded in the school health records (12 cases). Then, the students suspected of DCD were identified by the MABC- 2 movement test; finally, 56 volunteers with DCD were invited to participate in the research $[19,20]$. Next, the RT test, including visual, auditory, and tactile reaction time was performed by all participating students who were divided into 4 groups of 14 subjects.
The study subjects participated in the pretest; they were requested to touch the keys 1, 2, 3, and 4 based on the pattern provided by the specified time on the tablet monitor with a 12-inch screen to measure the sequential timing. The task was initially explained to the study subjects. Moreover, they conducted 5-10 repetitions to get acquainted with the test. The timer software designed by the researcher was similar to the one used by Ferguson et al. with changes in the shape of the keys, i.e., designed on the tablet (touch) where the screen touch provided a pattern of timing. The subject started the task by lifting their finger from the number one key and pressed the next keys with the specified timing. The information was recorded by touching the number 4 key. The collected results indicated that children with DCD act poorer and encounter challenges at a higher time intersection due to changing the brain processing system $[2,19]$.

Like Blais et al.'s task, the timing of this task was set with a slight change and with time sequences of 1500, 1500,400 , and $400 \mathrm{~m} / \mathrm{s}$ for keys 1 to 4 and time sequences of 150,150, and $100 \mathrm{~m} / \mathrm{s}$ for key distances 1 to 2,2 to 3 , and 3 to 4 . To calculate the time, the subjects should have lifted their fingers upon touching the start key and observing the pattern. Then, they should have touched the keys 1, 2, 3, and 4 based on the specified time. One attempt ended by touching key 4 . In the next step, the time stopped and the relative and absolute timing errors were recorded. Two sections of 5 attempts including warm-up and main run were presented for each test. Subsequently, the executive protocol was started and each of the 4 groups practiced in 10 blocks of 5 attempts; the auditory stimulation group concurrently received its accepted pattern with visual observing with the rhythmic auditory beat (140 BPM proportional \& continuous).

The SAS group received and practiced the sound of "continuous beep" as a presentation in addition to observing vision [20]. Control group 1 was the only recipient of the simple visual pattern. Control group 2 performed no exercise. Moreover, the other subjects spent time as usual under the supervision of instructors during the training. Consequently, all the subjects participated in the acquisition test with the same quality as the pre-test. Furthermore, the retention and transfer tests were performed 48 hours and 10 minutes later, respectively, with change in the specified times (1650, $1450,200, \& 200$ with sequences of 200, 150, \& 200 between the keys). Finally, the study subjects' relative and absolute error percentages were recorded. 


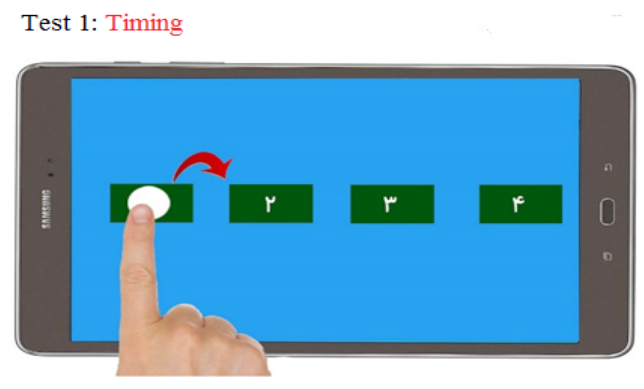

Figure 1. Instruments used for sequential timing tasks

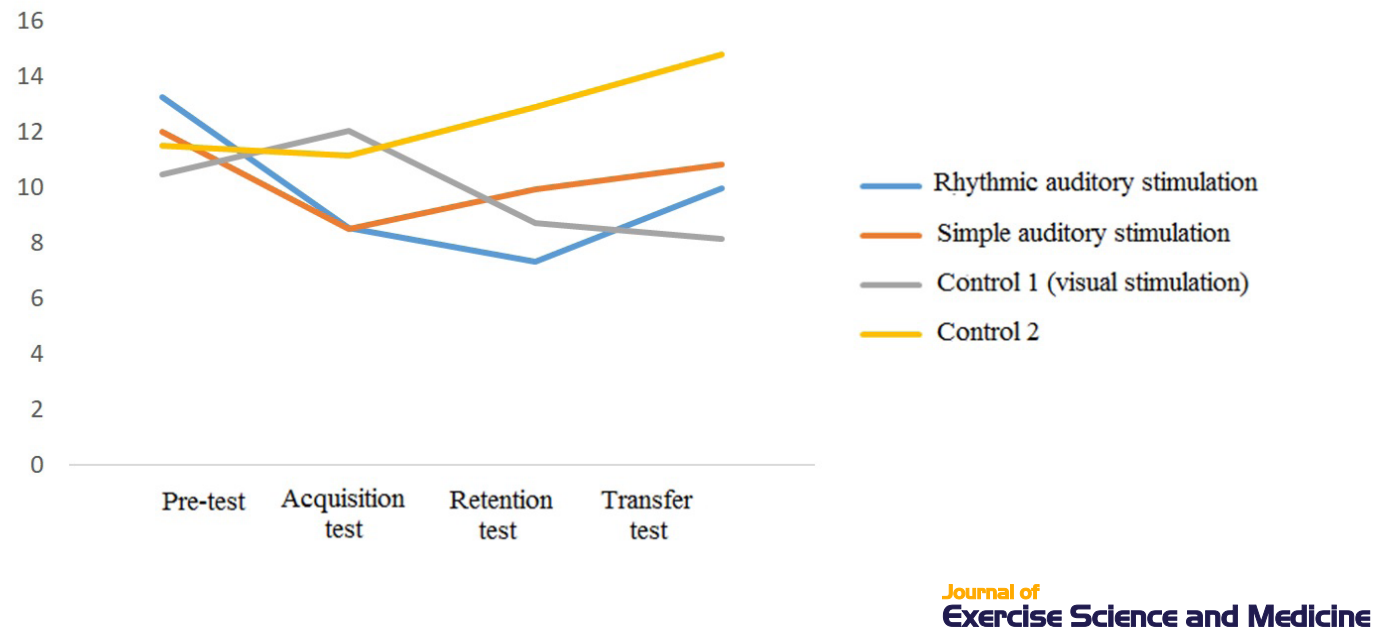

Figure 2. Relative timing changes (error percentage) in the sequential motor task from pretest to transfer test in the study groups

The obtained data were analyzed by SPSS. All examined variables were reported using descriptive statistics, such as mean and standard deviation. Shapiro-Wilk's test was used for assessing the normality of data. The Analysis of Covariance (ANCOVA) was used to compare the relative and absolute timings of the rhythmic and simple auditory stimulation groups, as well as the control groups 1 (visual) and 2 in the acquisition test. Additionally, one-way Analysis of Variance (ANOVA) was used to compare the relative timing of rhythmic and simple auditory stimulation groups, as well as the control groups 1 (visual) and 2 in retention and transfer tests. The significance level was set at $\mathrm{P}<0.05$.

\section{Results}

Table 1 indicates the descriptive information (mean \& standard deviation) related to the individual characteristics of the subjects by the study groups.

As illustrated in Figures 1 and 2, the changes in the relative timing variable (error percentage) of a sequential motor task in the RSA group from pretest to retention test followed a decreasing trend and slightly increased in the transfer test. However, the changes in the transfer

Table 1. Descriptive statistics on the individual characteristics of the subjects

\begin{tabular}{|c|c|c|c|c|c|}
\hline \multirow{2}{*}{ Variables } & \multirow{2}{*}{ Numbers } & \multicolumn{4}{|c|}{ Mean \pm SD } \\
\hline & & $\begin{array}{l}\text { Rhythmic Auditory } \\
\text { Stimulation }\end{array}$ & Simple Auditory Stimulation & $\begin{array}{c}\text { Visual Stimulation } \\
\text { Group }\end{array}$ & Control Group \\
\hline Age & 42 & $10.9 \pm 1.7$ & $9.80 \pm 1.3$ & $10.5 \pm 1.9$ & $10.75 \pm 1.2$ \\
\hline RT & 42 & $0.475 \pm 1.2$ & $0.463 \pm 1.1$ & $0.483 \pm 1.7$ & $0.466 \pm 1.5$ \\
\hline $\begin{array}{l}\text { Intelligence } \\
\text { Quotient }\end{array}$ & 42 & $97 \pm 1.5$ & $99 \pm 1.2$ & $95 \pm 1.6$ & $97 \pm 1.4$ \\
\hline
\end{tabular}




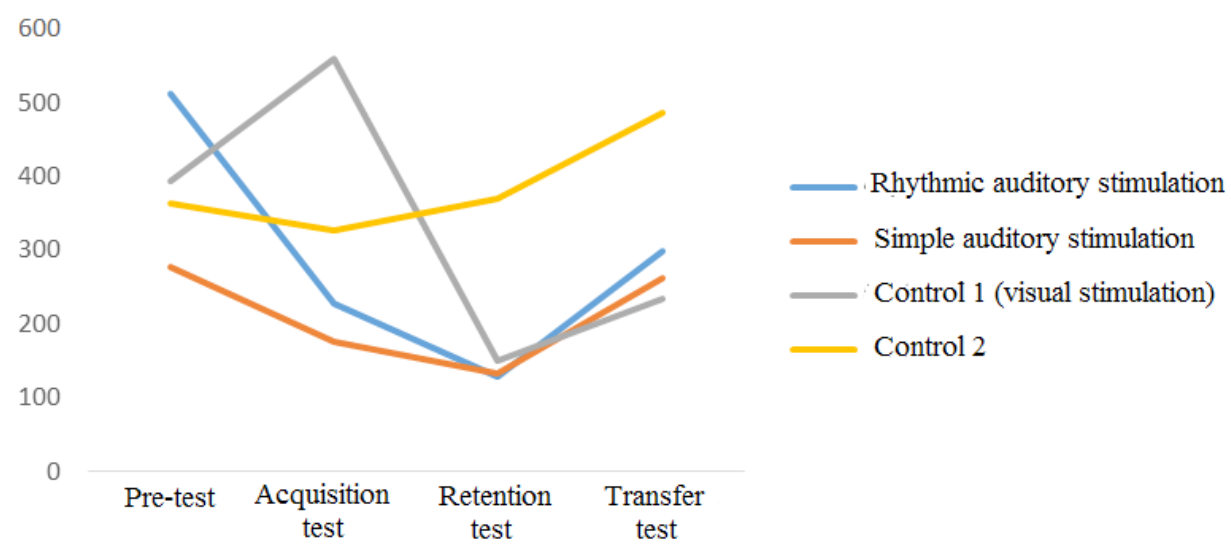

Figure 3. Absolute timing changes (errors) in sequential motor tasks from pretest to transfer test

test remained lower than those of the pretest. A similar pattern was observed in the SAS group and the control group 2 from the pretest to the transfer test. However, the relative timing error percentage of the SAS group in the retention and transfer test was lower than that of the control group 2. The visual stimulation group (control 1) provided an interesting pattern from pretest to transfer test; the percentage of relative timing error of this group in the acquisition test was higher than that of the pretest. However, the error percentage decreases in the retention test were lower than that of all of the groups in the transfer test.

As illustrated in Figure 3, changes in the absolute timing variable (error) of a sequential motor task in the rhythmic auditory stimulation group from pretest to retention test adopted a decreasing trend and increase slightly in the transfer test. However, the changes in the transfer test remained lower than those of the pretest. A similar pattern was detected in the SAS group and the control group 2 from the pretest to the transfer test. However, the absolute timing error of the SAS Group in the acquisition, retention, and transfer tests was lower than that of the control group 2. The visual stimulation group (control 1) presented an interesting pattern from pretest to transfer test so that the absolute timing error of this group in the acquisition test was more than that of the pretest. However, the error was reduced in the retention test to the level of rhythmic and SAS groups and was lower than that of all of the groups in the transfer test.

The relevant results indicated the normal distribution of data $(P>0.05)$. Based on the relationship between the dependent (posttest scores) and independent (pretest scores) variables, by drawing the distribution diagram and the best fit line, there was a linear relationship between the dependent and independent variables in all research groups, indicating the hypothesis of linearity in the covariance test. The hypothesis related to the homogeneity of regression slopes for performing ANCOVA was established. This is because the interaction effect of the group and independent variable was not statistically significant $(P=0.56)$. As per Table 2, the pretest effect, as a covariate (independent variable) in the absolute timing variables was not statistically significant $(P>0.05)$. How-

Table 2. ANCOVA data to compare the relative and absolute timings

\begin{tabular}{|c|c|c|c|c|c|c|c|}
\hline Variables & Resources & Total Squares & df & Mean Squares & $\mathbf{F}$ & $\mathbf{P}$ & Effect Size \\
\hline \multirow{3}{*}{ Relative timing } & Pretest effect & 79.26 & 1 & 79.26 & 10.62 & $0.002^{*}$ & 0.17 \\
\hline & Group & 183.98 & 3 & 61.33 & 8.22 & $0.0001^{*}$ & 0.32 \\
\hline & Error & 380.46 & 51 & 7.46 & & & \\
\hline \multirow{3}{*}{ Absolute timing } & Pretest effect & 1707.36 & 1 & 1707.36 & 0.27 & 0.6 & 0.005 \\
\hline & Group & 1214707.22 & 3 & 40492.40 & 65.63 & $0.0001^{*}$ & 0.79 \\
\hline & Error & 314621.20 & 1 & 6169.04 & & & \\
\hline
\end{tabular}


Table 3. Results of one-way ANOVA to compare the relative timing

\begin{tabular}{|c|c|c|c|c|c|c|}
\hline Type of Test & Resources & Total Squares & df & Mean Squares & $\mathbf{F}$ & $\mathbf{P}$ \\
\hline \multirow{3}{*}{ Retention test } & Between groups & 237.13 & 3 & 79.04 & 10.32 & $0.0001^{*}$ \\
\hline & Within group & 398.21 & 52 & 7.65 & & \\
\hline & total & 635.34 & 55 & & & \\
\hline \multirow{3}{*}{ Transfer test } & Between groups & 328.91 & 3 & 109.63 & 8.05 & $0.0001^{*}$ \\
\hline & Within group & 707.95 & 52 & 13.61 & & \\
\hline & total & 1036.86 & 55 & & & \\
\hline
\end{tabular}

ever, the pretest effect in the relative timing variable was statistically significant $(P \leq 0.05)$. In addition, the effect of group in relative and absolute timing variables in the acquisition test was statistically significant $(P \leq 0.05)$.

Based on Table 2, the group effect in the acquisition test for the relative timing variable was statistically significant. Therefore, the Bonferroni post hoc test was used to compare the two groups. The relevant results indicated a significant relationship between the rhythmic auditory stimulation group and visual stimulation $(P=0.001)$ and the control group $(P=0.02)$. Furthermore, a significant relationship was reported between the SAS group and visual stimulation $(P=0.002)$ in the relative timing variable. However, no significant difference was observed between the rhythmic auditory stimulation group and the SAS group ( $P>0.05)$, the SAS group and the control group $(P>0.05)$, and the visual stimulation group and the control group ( $P>0.05$ ). Furthermore, the effect of the group in the acquisition test for the absolute timing variable was statistically significant. Thus, the Bonferroni post hoc test was used to compare the two groups. Based on the obtained results, a significant difference was reported between the visual stimulation group and the control group ( $\mathrm{P}=0.0001)$, rhythmic auditory stimulation group $(\mathrm{P}=0.0001)$, simple auditory group ( $P=0.0001)$, as well as between the control group and SAS group $(\mathrm{P}=0.0001)$ in absolute timing. Besides, no significant difference was observed between the rhythmic auditory stimulation group, SAS group, and control group in absolute timing ( $P>0.05)$.

The results indicated that the hypothesis related to the homogeneity of variance in the relative timing variable was established in the retention and transfer tests $(P=0.55 ; P=0.53)$; however, the hypothesis related to the homogeneity of variance in the absolute timing variable was not established in the retention and transfer tests $(P=0.001 ; P=0.001)$. Thus, the Welch and BrownForsythe tests were used to examine the differences between the research groups.

As represented in Table 3, the between-group effects for the relative and absolute timing variables in the retention and transfer tests were statistically significant $(P \leq 0.05)$.

As per Table 3, the between-group effects for the relative timing variables were significant in the retention and the transfer tests. Therefore, Tukey's post hoc test was used to compare the two groups. In addition, a significant difference was reported between the control group 2 and rhythmic and SAS groups $(P=0.006 ; P=0.03)$,

Table 4. Welch and Brown-Forsythe tests data to compare the absolute timing

\begin{tabular}{cccccc}
\hline Variables & Tests & Statistics & df 1 & df 2 & P \\
\hline \multirow{2}{*}{ Retention test } & Welch & 18.19 & 3 & 27.03 & $0.0001^{*}$ \\
& Brown-Forsythe & 37.5 & 4 & 27 & $0.0001^{*}$ \\
Transfer test & Welch & 36.8 & 3 & 27.08 & $0.0001^{*}$ \\
& Brown-Forsythe & 22.04 & 3 & 23.2 & $0.0001^{*}$ \\
\hline
\end{tabular}


and the control group $1(\mathrm{P}=0.001)$ in the relative timing variable. However, no significant difference was observed between the relative timing of the rhythmic and SAS groups and the visual stimulation one $(P>0.05)$.

As indicated in Table 4, the Welch and Brown-Forsythe tests were significant for the absolute timing variable in the retention and transfer tests $(P \leq 0.05)$.

As presented in Table 4, the Welch and Brown-Forsythe tests were significant for the absolute timing variable in the retention and transfer tests. Thus, Tukey's post hoc test was used to compare the two groups. Accordingly, a significant difference was observed between the control group 2 and rhythmic and simple auditory stimulation groups, and the visual stimulation group $(\mathrm{P}=0.0001)$ in the absolute timing variable. However, no significant difference was reported between the rhythmic and simple auditory stimulation groups, and visual stimulation groups ( $P>0.05)$.

\section{Discussion}

During the timing tasks, the concept of frequency sequence or implementation period can occur at latent levels of auditory perception; they allow the "brain to be aware of the map and scale in time parameters from flexible positions" according to the exact reference distance. The profound effect of rhythm on the motor system indicates that time is considered a vital element of musical communication in motor behaviors although the exact biological process of rhythmic coordination remains unclear. Some studies supported that different aspects of processing time information can be attributed to the neocortex, basal ganglia, cerebellum, and thalamus [21, 22]. Based on the results, the rhythmic and simple auditory stimulation groups, as well as the control group 1 (visual stimulation) encountered declined relative timing error, indicating the facilitator effects of the auditory and visual stimulations in performing motor timing skills in the children with DCD; leading to rapid cerebellar involvement to encode the sequences accurately, and production of special circuits in the brain to represent the sequence structure of the chain timing [23].

Grube et al. (2010) reported that children with DCD act poorly in a visual rhythmic movement [23]. Accordingly, they should move their finger in a timed rhythm without stability and continuity of coordinationMoreover, he indicated that the auditory beat model can be useful for instant sensory-motor coordination to control actively [22]. Based on other evidence, perceiving the auditory timing and motor timing interact and form a specific pattern in the brain in each metronome-based period [23]. Based on the findings, training with rhythmic auditory stimulation led to a relative reduction of error compared to the practice of other groups due to the smaller metronome continuity (beat) of the auditory stimulation based on chain shocks. In addition, Maes et al. assessed two types of auditory stimulations called cohesive (continuous) and discrete (discontinuous) in a sequential task; they concluded that continuous auditory information is unnecessary.

It is regarded as an additional burden for working memory when the finger is removed from the keys to touch the next key. Therefore, the error of motor timing of continuous auditory stimulation was more than discrete auditory one [24]. Maes et al. used normal children in his study, while the subjects had DCD. Additionally, the type of auditory stimulation used in this study was different from the one applied in his study. This is because a rhythmic auditory stimulation was used with a subtle auditory beat every $25 \mathrm{~m} / \mathrm{s}$ and an original auditory beat in every $50 \mathrm{~m} / \mathrm{s}$ (so-called big drum). Besides, it can facilitate the subjects' auditory perception since the brain uses a distance timer to generate rhythmic responses which can be existed differently (cyclically) at regular beats as a useful indicator and help judge temporal changes [25]. Gerritsen (2009) evaluated individuals with cerebellar disorders in motor planning and adaptation to the situation [26]. They argued that these patients require external ear symptoms for motor coordination.

Based on the findings, there may be a direct relationship between auditory and motor structures for rehabilitation strategies in movement disorders. Furthermore, the temporal information can be useful for the motor system through the auditory system to extract functional change even in the presence of cerebellar injury. This is because the ability to coordinate remains intact, although the children with DCD usually represent a lack of coordination and movement timing over time. Thus, they coordinate with external signs, like auditory stimulation more accurately over time [27]. Moreover, the relative error between the rhythmic and simple auditory stimulations was not significantly different; it may be due to the lack of working memory and cognitive information of children with DCD.

The working memory is among the influential factors in the results considering the task time, i.e., more than one second. In addition, some studies indicated that timing at intervals of less than one second for children with DCD is primarily processed by subcortical networks, followed by basal ganglia and cerebellum; however, at intervals of more than one second, the processing relies 
primarily on the motor cortex and then on other cognitive functions, such as working memory, attention, and cognitive control [2]. In the present study, practicing the auditory beat coincided with presenting visual stimulations. Besides, the visual pattern was presented in two groups with rhythmic and simple auditory stimulations. Regardless of the type of the stimulation, including visual and auditory, the number of regular and sequential intervals of stimulations $(<500 \mathrm{~m} / \mathrm{s}$ ) more accurately affected timing processing, compared to their uniform presentation; even more close distances (vision) in a sequence help improve the system [28].

Generally, the brain recognizes auditory conditions more accurately than visual conditions [29]. In this study, the statistics related to the absolute timing error in control group 1 (visual stimulation) presented a significant effect, compared to the other groups. This is probably due to a greater number of close distances (vision) in a sequence, which help improve the system by giving more information to the memory [30]. MotorVisual coordination (performance) was better in continuous arrows; however, motor-auditory coordination was better in the discrete ones [14]. The auditory stimulation groups were observed with greater absolute error in the transition phase. This was probably because the learning of the whole sequential chain is related to cognitive and memory problems; it generally decreases slightly, because children and adolescents with DCD undergo working memory loss. Furthermore, the auditory stimulation before the transfer test represents a reduction in relative and absolute errors.

Morillon and Baillet believed that paying attention to predicting auditory timing is regarded as the source of sequential motor tasks [13]. Thus, the main information provided by the motor system includes the sensory processing of temporal content information like the audible beat intervals. Accordingly, the core of the motor system is based on supporting the timing and perceiving the time in presented stimulations [13]. However, Morillon and Baillet paid no attention to the type of stimulation and the lack of examining the quality and rhythm of the auditory stimulation was among the effective factors in analyzing the results.

Blais et al. compared the audio-visual stimulations between healthy children and children with DCD. They declared that the second group had relative learning in a rhythmic auditory rhythm, despite the weaker general performance. In addition, the accuracy and stability of a sensory-motor sequence increased with rhythmic auditory and visual stimulations in normal children and those with
DCD. However, the children with DCD represented more errors when the auditory stimulation was removed [20].

\section{Conclusion}

Based on the present study results, the children and adolescents with DCD resist change and their information processing speed in data transfer is slow. This is because motor learning problems, motor planning, adaptation to change, automation, sequence of movements, use of feedback, and prediction are considered as their obvious flaws. Additionally, internal modeling defects are evident among the children and adolescents with DCD, reflecting defects in inverse and forward models obtained for correction by comparing real and predicted results, which are poor in these children. Finally, there was no significant difference between rhythmic and simple auditory stimulations, although it seems that the first stimulation affects more than the second one.

A limitation of this study was no specific test for musical aptitude (familiarity with rhythm and multiplication) for these students to be evaluated. This was undoubtedly influential in the results. Another limitation was the lack of measuring the effect of the rhythmic auditory pattern on the brain for scheduling the test. It could be considered in future research. Furthermore, it is suggested that this type of task be evaluated with a rhythmic auditory stimulus according to the type of stimulus musical instrument. This is because the effect of sound waves on the brain may be different. Moreover, it is suggested that this test be performed by changing the size of the keys and monitor as well. This is because due to the delay in the sensory feedback of these students, the larger keys may lead to a more appropriate response.

\section{Ethical Considerations}

\section{Compliance with ethical guidelines}

All the Ethical Criteria of the Bio-Research Ethics Committee of the Ferdowsi University of Mashhad were observed in this study (Ethics Code: ID IR.UM.REC.1398.030).

\section{Funding}

This research did not receive any grant from funding agencies in the public, commercial, or non-profit sectors.

\section{Authors' contributions}

All authors equally contributed to preparing this article. 


\section{Conflict of interest}

The authors declared no conflict of interest.

\section{References}

[1] Adams IL. Predictive motor control in children with Developmental Coordination Disorder (DCD): Mechanisms and intervention. Nijmegen: Radboud University; 2018. https://repository.ubn.ru.nl/ handle/2066/178598

[2] Trainor L, Chang A, Cairney J, Li YC. Is auditory perceptual timing a core deficit of developmental coordination disorder? Annals of the New York Academy of Sciences. 2018; 1423(1):30-9. [DOI:10.1111/ nyas.13701] [PMID] [PMCID]

[3] Wilson PH, Smits-Engelsman B, Caeyenberghs K, Steenbergen B, Sugden D, Clark J, et al. Cognitive and neuroimaging findings in developmental coordination disorder: New insights from a systematic review of recent research. Developmental Medicine and Child Neurology. 2017; 59(11):1117-29. [DOI:10.1111/dmcn.13530] [PMID]

[4] Puyjarinet F, Begel V, Lopez R, Dellacherie D, Dalla Bella S. Children and adults with Attention-Deficit/Hyperactivity Disorder cannot move to the beat. Scientific Reports. 2017; 7(1):11550. [DOI:10.1038/s41598-017-11295-w] [PMID] [PMCID]

[5] Hyde C, Fuelscher I, Williams J, Lum JAG, He J, Barhoun P, et al. Corticospinal excitability during motor imagery is reduced in young adults with developmental coordination disorder. Research in Developmental Disabilities. 2018; 72:214-24. [DOI:10.1016/j. ridd.2017.11.009] [PMID]

[6] Rosenblum S, Regev N. Timing abilities among children with Developmental Coordination Disorders (DCD) in comparison to children with typical development. Research in Developmental Disabilities. 2013; 34(1):218-27. DOI:10.1016/j.ridd.2012.07.011] [PMID]

[7] Wilson PH, Ruddock S, Smits-Engelsman B, Polatajko H, Blank R. Understanding performance deficits in developmental coordination disorder: A meta-analysis of recent research. Developmental Medicine and Child Neurology. 2013; 55(3):217-28. [DOI:10.1111/j.14698749.2012.04436.x] [PMID]

[8] Lui KK, Nunez MD, Cassidy JM, Vandekerckhove J, Cramer SC, Srinivasan $\mathrm{R}$. Timing of readiness potentials reflect a decision-making process in the human brain. Computational Brain \& Behavior. 2018. Preprint. [DOI:10.1101/338806]

[9] Adams ILJ, Lust JM, Wilson PH, Steenbergen B. Compromised mo tor control in children with DCD: A deficit in the internal model? A systematic review. Neuroscience and Biobehavioral Reviews. 2014; 47:225-44. [DOI:10.1016/j.neubiorev.2014.08.011] [PMID]

[10] Debrabant J, Vingerhoets $G$, Van Waelvelde H, Leemans A, Taymans $\mathrm{T}$, Caeyenberghs $\mathrm{K}$. Brain connectomics of visual-motor deficits in children with developmental coordination disorder. The Journal of Pediatrics. 2016; 169:21-7. [DOI:10.1016/j.jpeds.2015.09.069] [PMID]

[11] Slater JL, Tate MC. Timing Deficits in ADHD: Insights from the neuroscience of musical rhythm. Frontiers in Computational Neuroscience. 2018; 12:51. [DOI:10.3389/fncom.2018.00051] [PMID] [PMCID]
[12] Patel $A D$, Iversen JR. The evolutionary neuroscience of musical beat perception: The Action Simulation for Auditory Prediction (ASAP) hypothesis. Frontiers in Systems Neuroscience. 2014; 8:57. [DOI:10.3389/fnsys.2014.00057] [PMID] [PMCID]

[13] Morillon B, Baillet S. Motor origin of temporal predictions in auditory attention. Proceedings of the National Academy of Sciences of the United States of America. 2017; 114(42):E8913-21. [DOI:10.1073/pnas.1705373114] [PMID] [PMCID]

[14] Hove MJ, Fairhurst MT, Kotz SA, Keller PE. Synchronizing with auditory and visual rhythms: An fMRI assessment of modality differences and modality appropriateness. Neuroimage. 2013; 67:313-21. [DOI:10.1016/j.neuroimage.2012.11.032] [PMID]

[15] Kral A. Auditory critical periods: A review from system's perspective. Neuroscience. 2013; 247:117-33. [DOI:10.1016/j.neuroscience.2013.05.021] [PMID]

[16] Varlet M, Marin L, Issartel J, Schmidt RC, Bardy BG. Continuity of visual and auditory rhythms influences sensorimotor coordination. PLoS One. 2012; 7(9):e44082. [DOI:10.1371/journal.pone.0044082] [PMID] [PMCID]

[17] Edwards WH. Motor learning and control: From theory to practice. Boston: Cengage Learning; 2010. https://books.google.com/ books?id=qeYHAAAAQBAJ\&dq

[18] Cantin N, Ryan J, Polatajko HJ. Impact of task difficulty and motor ability on visual-motor task performance of children with and without developmental coordination disorder. Human Movement Science. 2014; 34:217-32. [DOI:10.1016/j.humov.2014.02.006] [PMID]

[19] Ferguson GD, Duysens J, Smits-Engelsman BCM. Children with Developmental Coordination Disorder are deficient in a visuo-manual tracking task requiring predictive control. Neuroscience. 2015; 286:13-26. [DOI:10.1016/j.neuroscience.2014.11.032] [PMID]

[20] Blais M, Chauveau N, Biotteau M, Maziero S, Jucla M, Albaret J-M, et al. Cortical brain structure predicts memory of auditory vs visual rhythmic sequences in Children with and without Developmental Coordination Disorder (DCD). 2015; 1:1. https://tonic.inserm.fr/wpcontent/uploads/2019/09/Poster_Synchro_acaps.pdf

[21] Su YH. Audiovisual beat induction in complex auditory rhythms: Point-light figure movement as an effective visual beat. Acta Psychologica. 2014; 151(0):40-50 [DOI:10.1016/j.actpsy.2014.05.016] [PMID]

[22] de Oliveira llanz S, da Silva Oliveira D, de Azevedo Guendler J, Rocha M, Sarinho SW Effectiveness of motor intervention on children with Developmental Coordination Disorder (DCD): A systematic review. Journal of Physical Education and Sport Management. 2017; 8(3):32-40. [DOI:10.5897/JPESM2017.0291]

[23] Grube M, Cooper FE, Chinnery PF, Griffiths TD. Dissociation of duration-based and beat-based auditory timing in cerebellar degeneration. Proceedings of the National Academy of Sciences. 2010 107(25):11597-601. [DOI:10.1073/pnas.0910473107] [PMID] [PMCID]

[24] Maes PJ, Giacofci M, Leman M. Auditory and motor contributions to the timing of melodies under cognitive load. Journal of Experimental Psychology Human Perception \& Performance. 2015; 41(5):1336-52. [DOI:10.1037/xhp0000085] [PMID]

[25] Teki S, Grube M, Kumar S, Griffiths TD. Distinct neural substrates of duration-based and beat-based auditory timing. The Journal of Neuroscience. 2011; 31(10):3805-12. [DOI:10.1523/JNEUROSCI.5561-10.2011] [PMID] [PMCID] 
[26] Gerritsen J. A review of research done on Tomatis Auditory Stimulation. 2009. https://www.semanticscholar.org/paper/AReview-of-Research-done-on-34dbf

[27] Colvin A, Gough M. Individual employment rights arbitration in the U.S.: Actors and outcomes. Academy of Management. 2013; 2013:1. [DOI:10.5465/ambpp.2013.250]

[28] Drake C, Botte MC. Tempo sensitivity in auditory sequences: Evidence for a multiple-look model. Perception \& Psychophysics. 1993; 54(3):277-86. [DOI:10.3758/BF03205262] [PMID]

[29] Grahn JA. See what I hear? Beat perception in auditory and visual rhythms. Experimental Brain Research. 2012; 220(1):51-61. [DOI:10.1007/s00221-012-3114-8] [PMID]

[30] Adams IL, Lust JM, Steenbergen B. Development of motor imagery ability in children with developmental coordination disorder: A goal-directed pointing task. British Journal of Psychology. 2018; 109(2):187-203. [DOI:10.1111/bjop.12274] [PMID] 